\title{
DENGUE FEVER OUTBREAK IN TWIN CITIES; A TERTIARY CARE CENTER EXPERIENCE
}

\author{
Taimoor Ashraf Khan, Shazia Nisar*, Muhammad Mahad Qureshi**, Muhammad Israr**, Muhammad Samiullah
}

Headquarter Ghazaband Scouts Frontier Corps Balochistan, *Pak Emirates Military Hospital/National University of Medical Sciences (NUMS) Rawalpindi Pakistan, ${ }^{* *} 01$ Mountain Medical Battalion, Azad Jammu \& Kashmir, Pakistan, ${ }^{* * *}$ Force Command Northern Areas, Pakistan

\section{ABSTRACT}

Objective: To characterize the clinical presentations and laboratory markers (as disease predictors and markers) and severity of disease in patients admitted with dengue fever.

Study Design: Prospective observational study.

Place and Duration of Study: Department of Infectious Diseases, Pak Emirates Military Hospital Rawalpindi, from May to Oct 2018

Methodology: In our study, we included the patients treated for dengue fever. Data were recorded on predesigned proforma and analyzed using Microsoft excel 365 and Social Package for Statistical Sciences version 23.

Results: A total of 160 patients were included in this study, all were males. All cases were positive for Nonstructural Protein 1 Antigen. Symptoms seen in abundance were fever with rigors, chills, backache, retro-orbital pain, nausea and vomiting. Manual platelet count at admission ranged from 15-396 $\times 10^{3}$ (mean $107.5 \pm 6.8 \times 10^{3}$ ). Platelet count on discharge ranged from 102-577 x $10^{3}$ (mean $207.9 \pm 95.4 \times 10^{3}$ ). A total of $34(21.25 \%)$ patients out of 160 confirmed and admitted dengue fever cases had Dengue Hemorrhagic Fever (Nonstructural Protein 1 Antigen positive, thrombocytopenia and leukopenia) and 2 patient went into dengue shock syndrome. The derangement in alanine aminotransferase and alkaline phosphatase levels were shown in $98(61.2 \%)$ and $62(31.2 \%)$ of patients. All these patients were immunoglobulin M and Nonstructural Protein 1 Antigen positive.

Conclusion: Raised serum alanine aminotransferase and alkaline phosphatase levels along with early positive Nonstructural Protein 1 Antigen is a marker of disease severity.

Keywords: Alanine aminotransferase, Alkaline phosphatase, Dengue fever, Dengue hemorrhagic fever, Liver enzymes, Nonstructural protein 1 antigen.

How to Cite This Article: Khan TA, Nisar S, Qureshi MM, Israr M, Samiullah M. Dengue Fever Outbreak in Twin Cities; A Tertiary Care Center Experience. Pak Armed Forces Med J 2021; 71(5): 1524-1528. doi: https://doi.org/10.51253/pafmj.v71i5.4165

This is an Open Access article distributed under the terms of the Creative Commons Attribution License (https://creativecommons.org/licenses/by-nc/4.0/), which permits unrestricted use, distribution, and reproduction in any medium, provided the original work is properly cited.

\section{INTRODUCTION}

Dengue is one of the most widely transmitted vector-borne diseases in the world. It has become a global problem since World war II, effecting over 110 countries. As per estimates, 390 million dengue cases are reported every year with over 24,000 cases resulting in death..$^{1,2}$

Dengue virus is from the Flavivirus family and belongs to the Flavivirus genus, comprising of predominantly 4 serotypes (i.e. DENV-1. DENV-2, DENV3 and DENV-4), with the most recent fifth serotype (DENV-5) discovered in October 2013. ${ }^{3}$ It spreads using the mosquito from the Aedes family as a vector, predominantly the Aedes aegypti. However, other mosquitos from the Aedes family have also been noted to act as the vectors which include Aedes albopictus, Aedes polynesiensis and Aedes scutellaris. Aedes aegypti is a

Correspondence: Dr Muhammad Mahad Qureshi, 01 Mountain Medical Battalion, Bagh Azad Jammu \& Kashmir, Pakistan

Received: 20 Apr 2020; revision received: 25 Oct 2020; accepted: 26 Oct 2020 daytime feeder with peak number of mosquito bites usually seen at early mornings and dusk. It usually infects individuals below the height of 1000 meters above sea level. ${ }^{3}$

Dengue typically begins as a sudden-onset high grade bi-modal (saddle back) fever, headache that is characterized by patient's description of pain behind the eyes (retroorbital pain), myalgias, arthralgias, rash and may or may not have thrombocytopenic purpuric rash. The disease is divided into 2 phases i.e. febrile and recovery phase, with another phase 'critical phase' usually seen in children, young adults or debilitated adults. Women who develop dengue while pregnant may be at a greater risk of low birth weight baby and miscarriage. $^{4}$

Dengue can be diagnosed on clinical findings coupled with evidence on laboratory investigations that include detection of Nonstructural Protein 1 (NS1) Antigen, IgM and IgG antibodies to the virus. The infection also causes a transient derangement in platelet number, Liver Functions Tests (LFTs); Alanine 
Aminotransfe-rase (ALT) and Alkaline Phosphatase (ALP) and PT/PTTK.5,6

In Pakistan, serological testing of Anti-Dengue viral antibodies i.e. IgM and IgG, are generally used for confirmation of diagnosis of dengue. However, more accurate tests such as Reverse Transcriptase Polymerase Chain Reaction (RT-PCR) and detection of Non-structurad Protein-1 (NS-1) have emerged as more reliable methods of early diagnosis of dengue. The lack of resources and infrastructure, however, has limited the use of RT-PCR and NS-1 to only resourceful tertiary care hospitals of Pakistan. ${ }^{7}$

NS-1, is one of the seven non-structurad proteins encoded by the single strand RNA genome of the virus. It is an important diagnostic marker for the disease since it can be detected from the first day of onset of infection, whereas, the IgM is detected usually after the $4^{\text {th }}$ day of onset of disease. ${ }^{8}$

Infection with dengue virus can lead to Dengue Fever (DF), Dengue Hemorrhagic Fever (DHF) or Dengue Shock Syndrome (DSS). An individual infected with one dengue serotype becomes immune to re-infection from the same serotype; however, a re-infection with a different serotype generates a strong immune response leading to DHF which can be life threatening. ${ }^{9}$

Keeping in mind the great burden of disease of dengue worldwide and in our country, we conducted a prospective study to characterize the clinical presentations and laboratory markers (as disease predictors and markers) and severity of disease in patients admitted with dengue fever.

\section{METHODOLOGY}

This was a prospective observational study carried out in the department of infectious diseases, Pak Emirates Military Hospital (PEMH), Rawalpindi. A total of total of 567 suspected patients of dengue fever were evaluated in outpatient department and 160 confirmed cases of dengue fever who received inpatient care in the study period were included in this study. Universal sampling technique was used for sampling and we included 160 consecutive indoor patients in our study who fulfilled inclusion/exclusion criteria. Study was conducted from May to October 2018 and data was collected for the patients admitted with dengue fever in dengue ward over a period of 6 months during the dengue outbreak in twin cities of Rawalpindi and Islamabad. Data including age, gender, residential area, marital status, presenting complaints with duration, physical examination findings, results of lab reports, treatment administered, and duration of hospitalization were recorded on predesigned proforma by a single investigator. The series of investigations were carried out at the time of admission and on each day of hospital stay, which included blood complete picture with manual platelet count, liver function tests (Serum total bilirubin, ALT, ALP), renal function tests (i.e. Serum urea, creatinine, potassium and sodium levels), urine routine examination, ICT Malaria, dengue IgM \& IgG serology.

Inclusion Criteria: Patients with clinical examination supporting dengue fever and positive NS1 Antigen test at the time of admission or during the hospital stay, were included in the study.

Exclusion Criteria: Patients with any other known cause of acute febrile illness, hematological disorder, and or chronic liver disease were excluded.

Strict inclusion and exclusion criteria were followed. All patients were admitted in isolated infectious diseases' ward. Those with hemodynamical instability and or any other danger signs were shifted to ward H.D.U or medical ITC for further management. Leucopenia was characterized by white cells count $<4 \times 10^{9} / \mathrm{L}$ $\&$ for leukocytosis it was $>10 \times 10^{9} / \mathrm{L}$. Normal reference range for ALT was up to $42 \mathrm{U} / \mathrm{L}$ and for ALP , normal reference range is $21-100 \mathrm{IU} / \mathrm{L}$. Acute kidney injury was labelled on the basis of KDIGO guidelines. ${ }^{10}$. All the laboratory tests were performed and reported by a single expert pathologist from the same hospital's laboratory.

Written informed consent was taken from all the participants. Ethical approval was sought from the hospital ethical committee and institutional review board of PEMH Rawalpindi.

Data were analyzed using Microsoft excel 365 and Social Package for Statistical Sciences (SPSS) version 23. Descriptive analysis frequencies and percentages were calculated for all the qualitative variables. Mean and standard deviation were calculated for quantitative variables.

\section{RESULTS}

A total of 160 patients were included in this study and all were of male gender. At the time of presentation, they were residing in different areas of Rawalpindi and Islamabad city. All patients were diagnosed with dengue fever for the first time in their medical history at this presentation or hospital stay. The age 
range was 11-63 years with mean age of $32.9 \pm 11.5$ years, shown in Table-I.

Mean duration of hospital stay was $5.5 \pm 2.1$ days (range 2-12 days). Presenting complaints and physical examination findings of the patients are given in TableII, III with frequencies and percentages.

Table-I: Age distribution.

\begin{tabular}{|c|c|c|}
\hline \multicolumn{2}{|c|}{ Age Group } & n (\%) \\
\hline \multicolumn{2}{|c|}{$11-22$ years } & $22(13.8)$ \\
\hline \multicolumn{2}{|c|}{ 23-32 years } & $72(45)$ \\
\hline \multicolumn{2}{|c|}{$33-42$ years } & $32(20)$ \\
\hline \multicolumn{2}{|c|}{$43-52$ years } & $24(15)$ \\
\hline \multicolumn{2}{|c|}{$53-62$ years } & $6(3.8)$ \\
\hline \multicolumn{2}{|c|}{$>62$ years } & $4(2.5)$ \\
\hline \multicolumn{3}{|c|}{ Table-II: Presenting complaints. } \\
\hline \multicolumn{2}{|c|}{ Presenting Complaint } & n (\%) \\
\hline \multirow{2}{*}{ Fever } & High Grade & $148(92.5)$ \\
\hline & Low Grade & $12(7.5)$ \\
\hline \multicolumn{2}{|l|}{ Rigors } & $116(72.5)$ \\
\hline \multicolumn{2}{|l|}{ Chills } & $128(80)$ \\
\hline \multicolumn{2}{|c|}{ Backache } & $134(83.7)$ \\
\hline \multicolumn{2}{|c|}{ Generalized Body Aches } & $84(52.5)$ \\
\hline \multicolumn{2}{|c|}{ Nausea } & $60(37.5)$ \\
\hline \multicolumn{2}{|c|}{ Vomiting } & $50(31.3)$ \\
\hline \multicolumn{2}{|c|}{ Retro-Orbital Pain } & $44(27.5)$ \\
\hline \multicolumn{2}{|c|}{ Cough } & $14(8.8)$ \\
\hline \multicolumn{2}{|c|}{ Burning Micturition } & $4(2.5)$ \\
\hline \multicolumn{2}{|c|}{ Sore Throat } & $32(20)$ \\
\hline \multicolumn{3}{|c|}{ Table-III: Physical examination findings. } \\
\hline \multicolumn{2}{|c|}{ Physical Examination } & n (\%) \\
\hline \multicolumn{2}{|c|}{ Hepatomegaly } & $4(2.5)$ \\
\hline \multicolumn{2}{|c|}{ Splenomegaly } & $16(10)$ \\
\hline \multicolumn{2}{|c|}{ Congested throat } & $50(31.3)$ \\
\hline Rash & & $12(7.5)$ \\
\hline Dehyd & & $36(22.5)$ \\
\hline Conjur & gestion & $8(5)$ \\
\hline Post-au & mphadenopathy & $6(3.8)$ \\
\hline Tourni & & $42(26.2)$ \\
\hline Table- & of platelets on a & and discharge. \\
\hline Platele & ission & \\
\hline Rang & & $\mathrm{n}(\%)$ \\
\hline $0-50$ & & $24(15)$ \\
\hline $51-10$ & & $60(37.5)$ \\
\hline $101-1$ & & $52(32.5)$ \\
\hline 151-2 & & $14(8.8)$ \\
\hline$>200$ & & $10(6.3)$ \\
\hline Platele & harge & \\
\hline Rang & & $\mathrm{n}(\%)$ \\
\hline $101-1$ & & $46(28.7)$ \\
\hline 151-2 & & $50(31.3)$ \\
\hline $201-2$ & & $22(13.8)$ \\
\hline $251-4$ & & $34(21.3)$ \\
\hline$>400$ & & $8(5)$ \\
\hline
\end{tabular}

Manual platelet count at admission ranged from $15-396 \times 10^{3}$ while mean was $107.5 \pm 6.8 \times 10^{3}$. Platelet count on discharge ranged from 102-577 x $10^{3}$ (mean $\left.207.9 \pm 95.4 \times 10^{3}\right)$. Range of platelets in groups shown in Table-IV.

Mean hematocrit at admission was $42 \pm 3.8 \%$ while $41 \pm 4.3 \%$ at discharge. Thirty four patients had leucopenia while only 4 had leukocytosis. Acute liver injury was reported as per the operational definition in 28 patients $(17.5 \%)$. The ALT levels were deranged in $98(61.2 \%)$ patients. In 48 patients ALT was $>2$ times, $>3$ times upper reference limit in 18 patients, $>4$ times in $4,>5$ times in 2 and $>6$ times of upper reference limit in 4 patients. All these cases were positive for IgM serology ALP was deranged in $62(31 \%)$ of the patients.

All patients were NS1 antigen positive while dengue serology has varying results i.e. IgM was positive in $68(42.5 \%), \operatorname{IgG}$ positive in $4(2.5 \%)$, Both IgM \& IgG positive in $2(1.3 \%)$ and in $86(53.3 \%)$ patients both IgM and IgG were negative. There was no clinical difference in severity of the patients with positive IgG. Four $(2.5 \%)$ patients had co-infection of malaria in which ICT malaria was positive.

A total of 34 patients out of 160 confirmed DF cases had DHF (NS1 antigen positive, thrombocytopenia and leukopenia) and only two patient went into DSS. No patient in our study population was expired and all were discharged home after indoor management at the hospital.

Table-V: Mean laboratory parameters.

\begin{tabular}{l|c|c}
\hline Laboratory Test & Mean Value \pm SD & Range \\
\hline Hemoglobin g/dl & $14.2 \pm 1 \mathrm{~g} / \mathrm{dl}$ & $12-16$ \\
\hline TLC $10^{9} / \mathrm{L}$ & $4.9 \pm 1.510^{9} / \mathrm{L}$ & $2-11$ \\
\hline ALT U/L & $76 \pm 8.1 \mathrm{U} / \mathrm{L}$ & $4-599$ \\
\hline ALP IU/L & $94 \pm 4.3 \mathrm{IU} / \mathrm{L}$ & $29-229$ \\
\hline Total Bilirubin $\mu \mathrm{mol} / \mathrm{L}$ & $10.9 \pm 5.1 \mu \mathrm{mol} / \mathrm{L}$ & $7-41$ \\
\hline Serum Urea $\mathrm{mmol} / \mathrm{L}$ & $4.3 \pm 1.2 \mathrm{mmol} / \mathrm{L}$ & $2-7.7$ \\
\hline Serum Creatinine $\mu \mathrm{mol} / \mathrm{L}$ & $97 \pm 2.1 \mu \mathrm{mol} / \mathrm{L}$ & $50-148$ \\
\hline
\end{tabular}

\section{DISCUSSION}

The first documented dengue infections in Pakistan date back to the years 1994-95. ${ }^{11}$ There have been 10 major dengue outbreaks ever since. In 2011, there were 50,000 reported cases in Lahore alone. It was followed by another major outbreak in KPK two years later in 2013 when 8546 cases were reported. In 2013 district Swat alone had 6000 cases reported out of which $69 \%$ were seen to be males. ${ }^{12,13}$ As one of the most common vectorborne diseases of Pakistan, dengue presents as a challenge to our health care setup owing to the lack of diagnostic facilities and due to the varying clinical presentation of disease. Thus, due to 
lack of surveillance and paucity of diagnostic facilities many cases of dengue go unreported every year. As per an epidemiological study conducted in the 1960's and 1980's, significant numbers of normal healthy population were found to be positive for dengue specific antibodies. ${ }^{14}$

Hepatic involvement in the course of dengue infection is seen in the form of elevated levels of aminotransferases. With more acute and abrupt rise seen in children, immunocompromised and in secondary dengue infections leading to DHF and DSS.15,16 Hence, an elevated level of aminotransferases can serve as an indicator of hepatic dysfunction which can be due to hepatotoxic drugs administered during the hospital stay and because of the virus itself.

In the current study, clinical features, diagnostic and laboratory tests of all patients who were admitted in Dengue Ward of PEMH in 2018, were studied. All patients admitted presented with fever as a predominant feature. Other symptoms seen in abundance were rigors, chills, backache, retro-orbital pain, nausea and vomiting. A study conducted in Peshawar (Pakistan) showed almost similar findings of fever, generalized weakness and vomiting being present in 100\%, 73\% and $43 \%$ percent of cases respectively. However, one stark difference was noted as the study showed $70 \%$ of cases having hepatomegaly in contrast to our study where only $2.5 \%$ of cases had hepatomegaly and $10 \%$ had splenomegaly upon presentation to the clinic. ${ }^{12}$ The prevalence of disease (38\%) predominantly in the age groups between 21-40 years was in correspondence to our study where $45 \%$ of patients admitted in dengue ward were in between the ages of 23-32 years.

Patients testing positive for NS1, along with signs and symptoms corresponding with the disease was a part of admission criteria to our dengue ward in PEMH. Dengue serology i.e. IgM and IgG, was also tested in patients presenting to our setup. All patients admitted in our dengue ward were seen to be NS1 positive at time of presentation or during stay. A similar finding was seen in a study undertaken in Malaysia, thus, highlighting the importance of NS1 as a marker of dengue in early phases of disease. ${ }^{1}$ A study conducted in Australia on travelers returning from dengue endemic areas showed similar diagnostic results with $33 \%$ ( $42 \%$ in our setup) being tested positive for IgM and NS1 both upon presentation. Two percent were seen to be positive for IgG and NS1 (2.5\% in our study) whereas, $4 \%$ were positive for IgG, IgM and NS1 (1.3\% in our study). ${ }^{17}$
Other laboratory tests conducted during patient stay in dengue ward showed findings like other studies conducted before. A study in Malaysia shows $80 \%$ of patients presenting with a low platelet count, mean WBC count as $3.73 \times 10^{3} / \mu \mathrm{l}$, mean $\mathrm{Hb}$ of $10.8 \mathrm{~g} / \mathrm{dl}$ and mean hematocrit at $38.9 \% .{ }^{1}$ In our study, however, hematocrit was tested at time of presentation and at time of discharge showing a mean of $42 \pm 3.2 \%$ and $41 \pm$ $4.3 \%$ respectively. We also observed a mean TLC count of $4.9 \pm 1.510^{9} / \mathrm{L}$ in our study with a total of $28(17 \%)$ patients showing leucopenia whereas, only a mere $2 \%$ showed reactive thrombocytosis. Lei et al, also shows majority of Dengue patients having leucopenia at time of presentation or during their stay at the hospital, with a reduction seen in absolute number of monocytes and neutrophils. ${ }^{18}$

Dengue patients were also seen to have derangement in renal function tests with the study quoted above showing a derangement in $23 \%$ of cases with a mean serum urea levels of $40.2 \mathrm{mg} / \mathrm{dl}$ and serum creatinine levels of $1.5 \mathrm{md} / \mathrm{dl} .{ }^{1}$ In our study, serum urea levels were seen to be fluctuating between $36-139 \mathrm{mg} /$ $\mathrm{dl}$ and serum creatinine levels between 50-148 $\mu \mathrm{mol} / \mathrm{L}$, with a mean of $4.3 \pm 1.2 \mathrm{mmol} / \mathrm{L}$ and $97 \pm 2.1 \mu \mathrm{mol} / \mathrm{L}$ respectively.

A stark difference is again shown in our study when trend in derangement of liver enzymes was studied. The derangement in ALT and ALP levels were shown in $61.2 \%$ and $31.2 \%$ of patients. All these patients were IgM and NS1 antigen positive. This is contrary to the results shown by Prakash et al, where ALT was deranged in $86 \%$ and ALP was deranged in 95\% patients. ${ }^{19}$ Kuo et al, reported $90 \%$ of ALP derangement in dengue patients. ${ }^{20}$ We assumed that low threshold for admission of the dengue fever patients for inpatient care is the cause for lesser severity of the disease and minimum possible incidence of complications including acute liver injury. Moreover, NS1 Antigen is a marker which rises early in the disease so it's quite a possibility that these liver enzymes don't usually get deranged in early phase of dengue fever. ${ }^{1}$

Strength of this study is that it has given the trends of severity and biochemical markers of the recent dengue outbreak in Rawalpindi and Islamabad.

\section{CONCLUSION}

Raised serum ALT and ALP along with early positive NS1 antigen is a marker of disease severity. Low threshold for admissions and inpatient care in a close monitoring environment of such patients can lead to successful outcomes and minimum complications. Careful clinical examination 
along with daily laboratory investigations including liver function tests and dengue NS1 antigen is invaluable in diagnosis and appropriate management of this disease.

\section{LIMITATION OF STUDY}

It was single center study with small sample size and included the patients taking inpatient care only. The study population in our study was entitled to the treatment and were only residing in one of these cities at the time of presentation and were not necessarily the permanent residents of these cities. Thus, they didn't represent the true population of the twin cities.

\section{Conflict of Interest: None.}

\section{Authors' Contribution}

TAK: Conception of design, data collection \& analysis, writing and editing of manuscript, SN: Conception of design, data collection \& analysis, writing and editing of manuscript, MMQ: Data collection \& analysis, writing and editing of manuscript, MI: Conception of design, data collection \& analysis, writing and editing of manuscript, MS: Data collection \& analysis, writing and editing of manuscript.

\section{REFERENCES}

1. Rashmi MV, Hamsaveena.Haematological and biochemical markers as predictors of dengue infection. Malays J Pathol 2015; 37(3): 247-251.

2. Ahmad H, Ullah I, Ahmad T. Outbreak investigation report of dengue fever during June-November 2014: A case study of district Swat, Pakistan. J Pak Med Assoc 2018; 68(2): 192-194.

3. Mustafa MS, Rasotgi V, Jain S, Gupta V. Discovery of fifth serotype of dengue virus (DENV-5): A new public health dilemma in dengue control. Med J Arm For Ind 2015; 71(1): 67-70.

4. Basurko C, Everhard S, Matheus S, Restrepo M, Hildéral H, Lambert $\mathrm{V}$, et al. A prospective matched study on symptomatic dengue in pregnancy. PLoS One 2018; 13(10): e0202005.

5. Hussain T, Jamal M, Rehman T, Andleeb S. Dengue: pathogenesis, prevention and treatment - a mini review. Adv Life Sci 2015; 2(3): 110-104.

6. Babaliche P, Doshi D. Catching dengue early: clinical features and laboratory markers of dengue virus infection. J Assoc Phys Ind 2015; 63(5): 38-41.
7. Afridi N, Ahmed S, Khan S, Ali N, Rauf S. early diagnosis of dengue virus infection in clinically suspected cases. Pak Armed Forces Med J 2016; 66(6): 837-840.

8. Chan HBY, How $\mathrm{CH}, \mathrm{Ng} \mathrm{CW}$. Definitive tests for dengue fever: when and which should I use?. Singapore Med J 2017; 58(11): 632-635.

9. Waggoner JJ, Balmaseda A, Gresh L, Sahoo MK, Montoya M, Wang $\mathrm{C}$, et al. Homotypic dengue virus reinfections in nicaraguan children. J Infect Dis 2016; 214(7): 986-993.

10. KDIGO Guidelines. Global Science. Local Change, [Internet] Available from url: https://kdigo.org/guidelines. [Accessed on Apr 27, 2019].

11. Shakoor MT, Ayub S, Ayub Z.Dengue fever: Pakistan's worst nightmare. WHO South East Asia J Public Health. 2012; 1(3): 229231.

12. Haroon M, Jan H, Faisal S, Ali N, Kamran M, Ullah F. Dengue outbreak in peshawar: clinical features and laboratory markers of dengue virus infection. J Infect Public Health 2019; 12(2): 258-262.

13. Akinsolu FT, de Paiva VN, Souza SS, Varga O. Patent landscape of neglected tropical diseases: an analysis of worldwide patent families. Global Health 2017; 14-13(1): 82.

14. Rasheed SB, Butlin RK, Boots M. A review of dengue as an emerging disease in Pakistan. Public Health 2013; 127(1): 11-17.

15. Souza LJ, Alves JG, Nogueira RM, Gicovate Neto C, Bastos DA, Siqueira EW, et al. Aminotransferase changes and acute hepatitis in patients with dengue fever: analysis of 1,585 cases. Braz J Infect Dis 2004; 8(2): 156-163.

16. Wiwanitkit V. Liver dysfunction in dengue infection, an analysis of the previously published thai cases. J Ayub Med Coll Abbott 2007; 19(1): 10-12.

17. Quinn EJ, Cheong AH, Calvert JK, Higgins G, Hahesy T, Gordon DL, Carr JM. Clinical features and laboratory findings of travelers returning to south australia with dengue virus infection. Trop Med Infect Dis 2018; 7-3(1): 6-10.

18. Lei HY, Yeh TM, Liu HS, Lin YS, Chen SH, Liu CC. Immunopathogenesis of dengue virus infection. J Biomed Sci 2001; 8(5): 377-388.

19. Parkash O, Almas A, Jafri SM, Hamid S, Akhtar J, Alishah H. Severity of acute hepatitis and its outcome in patients with dengue fever in a tertiary care hospital Karachi, Pakistan (South Asia). BMC Gastroenterol 2010; 7(10): 43-48.

20. Kuo CH, Tai DI, Chang-Chien CS, Lan CK, Chiou SS, Liaw YF. Liver biochemical tests and dengue fever. Am J Trop Med Hyg 1992; 47(3): 265-270. 